\title{
COVID-19 Pandemic: A Review
}

\author{
Mysore D Shylaja ${ }^{1}$, Jyoti Zingade ${ }^{2}$, Pavan K Gujjar ${ }^{3}$, Kailash M Attur ${ }^{4}$
}

\begin{abstract}
The World Health Organization (WHO) announced that the outbreak of the novel coronavirus (CoV) has constituted a public health emergency of international concern. Coronavirus belongs to the genus Coronavirus with its high mutation rate in the Coronaviridae. The epidemic of coronavirus disease 2019 (COVID-19) originating in Wuhan, China, has become a major public health challenge not only for China but also for countries around the world. Infection control measures are necessary to prevent the virus from spreading further and to help control the epidemic situation. For dental practices and hospitals in areas that are (potentially) affected by COVID-19, strict and effective infection control protocols are urgently needed.

Keywords: Coronavirus, COVID-19, Dental practice, Virology.

Journal of Oral Health and Community Dentistry (2020): 10.5005/jp-journals-10062-0081
\end{abstract}

\section{INTRODUCTION}

Coronaviruses (CoVs) are positive-stranded RNA viruses with the largest viral genome (27-33 kb). The virus particles are enveloped and carry extended spike proteins on the membrane surface, providing the typical crown-like structure (crown = corona). CoVs are known to infect mammals and birds. Genotypically, the CoVs can be subdivided into three groups. Group III viruses are found exclusively in birds, whereas viruses from groups I and II have mammals as their host. CoVs belong to the genus Coronavirus in the Coronaviridae family. ${ }^{1}$

All CoVs are pleomorphic RNA viruses characteristically containing crown-shaped peplomers with 80 to $160 \mathrm{~nm}$ in size. Recombination rates of CoVs are very high because of constantly developing transcription errors and RNA-dependent RNA polymerase (RdRP) jumps. ${ }^{2}$ With its high mutation rate, CoVs are zoonotic pathogens that are present in humans and various animals with a wide range of clinical features from asymptomatic course to requirement of hospitalization in the intensive care unit, causing infections in respiratory, gastrointestinal, hepatic, and neurologic systems. ${ }^{3}$

The virus was not considered as highly pathogenic for humans until they have been seen with the severe acute respiratory syndrome (SARS) in the Guangdong state of China for the first time in 2002 and 2003. ${ }^{4}$ Approximately 10 years after SARS, another highly pathogenic CoV, Middle East respiratory syndrome coronavirus (MERS-CoV) emerged in the Middle East countries. ${ }^{5}$ In December 2019 novel coronavirus (nCoV), which initiated another public health problem, was taught to have emerged in the Huanan Seafood Market, where livestock animals are also traded, in Wuhan State of Hubei Province in China and has been the focus of global attention due to a pneumonia epidemic of unknown cause. ${ }^{4}$

A primarily unknown pneumonia case was detected on December 12,2019, and possible influenza and other coronaviruses were ruled out by laboratory testing. Chinese authorities announced on January 7, 2020, that a new type of coronavirus ( $\mathrm{nCoV}$ ) was isolated. This virus was named COVID-19 by WHO on January $12,2020 .{ }^{6}$ Initially the infection transmitted probably as a zoonotic agent (from animal to human). The increase in the number of cases in Wuhan city and internationally after closing the market and evacuation of the cases in China have indicated a second
${ }^{1}$ Department of Oral Pathology, Narsinhbhai Dental College and Hospital, Visnagar, Gujarat, India

${ }^{2}$ Department of Oral Medicine and Radiology, MN DAV Dental College and Hospital, Solan, Himachal Pradesh, India

${ }^{3}$ Department of Oral Pathology and Microbiology, MN DAV Dental College and Hospital, Solan, Himachal Pradesh, India

${ }^{4}$ Department of Conservative Dentistry and Endodontics, Narsinhbhai Dental College and Hospital, Visnagar, Gujarat, India

Corresponding Author: Pavan K Gujjar, Department of Oral Pathology and Microbiology, MN DAV Dental College and Hospital, Solan, Himachal Pradesh, India, Phone: +91 8347060388, e-mail: jyopavan2612@gmail.com

How to cite this article: Shylaja MD, Zingade J, Gujjar PK, et al. COVID-19 Pandemic: A Review. J Oral Health Comm Dent 2020;14(3):110-112.

Source of support: Nil

Conflict of interest: None

transmission from human-to-human. ${ }^{4}$ The objective of this review article is to have a primary outlook about the disease, the ways of treatment, and prevention in the early stage of this outbreak.

\section{Epidemiology}

In December 2019, multiple pneumonia cases were clustered in Wuhan city and investigations for the source pointed toward Huanan Seafood Market as the origin. ${ }^{6}$ The first case of the COVID19 was discovered with unexplained pneumonia on December 12,2019 , and 27 viral pneumonia cases, with 7 being severe, were officially announced on December 31, 2019. ' On December 31, 2019, China notified the outbreak to the WHO, and on January 1, 2020, the Huanan Seafood Market was closed. On January 7, 2020, the virus was identified as a coronavirus that had $>95 \%$ homology with the bat coronavirus and $>70 \%$ similarity with the SARS-CoV. ${ }^{8}$ Environmental samples from the Huanan Seafood Market also tested positive, signifying that the virus originated from there. Similar to the SARS epidemic, this outbreak has occurred during the Spring Festival in China, which is the most famous traditional festival, during which nearly three billion people travel countrywide. This gave a favorable condition for the transmission of this highly 
contagious disease and severe difficulties in prevention and control which grew to become epidemic. ${ }^{9}$

The period of the Spring Festival of China was between January 17 and February 23 in 2003, when the SARS epidemic peaked, while the period of the festival was between January 10 and February 18 in 2020. Similarly, there was a rapid increase in COVID-19 cases during January 2020. Wuhan, the center of the epidemic with a 10 million population, is also an important center in the spring festival transportation network. The estimated number of travelers during the 2020 spring festival rose 1.7 folds when compared with the number traveled in 2003 and reached 3.11 billion from 1.82 billion. This large-scale travel traffic has also created favorable conditions for the spread of this difficult-tocontrol disease. ${ }^{4}$

\section{Pathogenesis}

The first step in virus infection is the interaction of sensitive human cells with spike protein. Genome encoding occurs after entering the cell and facilitates the expression of genes that encode accessory proteins, which advance the adaptation of CoVs to their human host. $^{10}$

Genome changes resulting from recombination, gene exchange, gene insertion, or deletion are frequent among CoVs, and this will take place in future outbreaks as in past epidemics. As a result of the studies, the CoV subfamily is rapidly expanding with new generation sequencing applications that improve the detection and definition of novel CoV species. According to the most recent classification of the International Committee on Taxonomy of Viruses (ICTV), there are four genera of 38 unique species. ${ }^{11}$

\section{Mode of Transmission}

Coronaviruses have been defined as a novel respiratory tract virus in the samples collected from the individuals who present symptoms of respiratory tract infection in 1962. This is a large family of viruses that are common in many different animal species, including camels, cattle, cats, and bats. ${ }^{11}$

Rarely, animal CoVs can infect humans and, as a result, may spread among humans during epidemics, such as MERS, SARS, and COVID-19. At the onset of major outbreaks caused by CoVs, palm cats have been proposed to be a natural reservoir of human CoVs for SARS and dromedary camels for MERS. ${ }^{12}$ However, more advanced virological and genetic studies have shown that bats are reservoir hosts of both SARS-CoV and MERS-CoV, and before these viruses spread to humans, they use the other responsible animals as intermediate hosts. ${ }^{3}$ Often, human-to-human transmission occurs with close contact. The transmission primarily occurs when an infected person sneezes and through the respiratory droplets produced just as the spread of influenza and other respiratory pathogens. These droplets can settle in the mouth or nasal mucosa and lungs of people with inhaled air. Currently, it remains unclear whether a person can be infected by COVID-19 by touching an infected surface or object and then touching their mouth, nose, or possibly eyes. ${ }^{13}$

Like most respiratory viruses, it is considered to be the most contagious when people are most symptomatic. However, cases, which were infected from an asymptomatic person in the prodromal period of COVID-19, were also reported. Sufficient data are not available on the infectiousness of the disease and research is ongoing. ${ }^{14}$

\section{Prevention}

Presently there are no approved treatments for this infection, and prevention is crucial. Several properties of this virus make prevention difficult, namely, nonspecific features of the disease, the infectivity even before the onset of symptoms in the incubation period, transmission from asymptomatic people, long incubation period, tropism for mucosal surfaces, such as the conjunctiva, prolonged duration of the illness and transmission even after clinical recovery. ${ }^{15}$

Isolation of confirmed or suspected cases with mild illness at home is recommended. The ventilation at home should be good with sunlight to allow for the destruction of the virus. Patients should be asked to wear a simple surgical mask and practice cough hygiene. Caregivers should be asked to wear a surgical mask when in the same room as the patient and use hand hygiene every 15 to 20 minutes. $^{16}$

The greatest risk in COVID-19 is transmission to healthcare workers. In the SARS outbreak of 2002, $21 \%$ of those affected were healthcare workers. Healthcare workers should be provided with fit-tested N95 respirators, protective suits, and goggles. Airborne transmission precautions should be taken during aerosol-generating procedures, such as intubation, suction, and tracheostomies. All contacts including healthcare workers should be monitored for the development of symptoms of COVID-19.15

At the community level, people should be asked to avoid crowded areas and postpone nonessential travel to places with ongoing transmission. They should be asked to practice cough hygiene by coughing in sleeve or tissue rather than hands and practice hand hygiene frequently every 15-20 minutes. Patients with respiratory symptoms should be asked to use surgical masks. ${ }^{17}$

\section{Precautions Taken for COVID-19 during General Practice for a Practitioner}

- Healthcare providers should take the travel history of all patients with respiratory symptoms, and any international travel in the past 2 weeks as well as contact with sick people who have traveled internationally.

- They should set up a system of triage of patients with respiratory illness in the outpatient department and give them a simple surgical mask to wear. They should use surgical masks themselves while examining such patients and practice hand hygiene frequently.

- Suspected cases should be referred to the governmentdesignated centers for isolation and testing.

- Patients admitted with severe pneumonia and acute respiratory distress syndrome should be evaluated for travel history and placed under contact and droplet isolation. Regular decontamination of surfaces should be done. They should be tested for etiology using multiplex PCR panels if logistics permit and if no pathogen is identified, refer to the samples for testing or SARS-CoV-2.

- All clinicians should keep themselves updated about recent developments including the global spread of the disease.

- Nonessential international travel should be avoided at this time.

\section{Management of Acute Dental Problems DURING COVID-19}

Emergency Dental Care and Management of Acute Dental Problems guidance publication describes modified management 
of commonly presenting oral conditions for use during the COVID-19 pandemic. It aims to encourage a consistent approach to the management of acute dental problems while recognizing the challenges that the COVID-19 pandemic presents for the provision of dental care. It can be used in conjunction with the health board or other local procedures that have been established for managing patients based on their COVID-19 status.

The management options presented here focus on dental triage, the relief of pain or infection, and the provision of care using remote consultation (i.e., by telephone or video call). Patients should only be referred for urgent dental care when there are severe or uncontrolled symptoms that they cannot manage themselves. It is essential to minimize the number of patients referred to designated urgent dental care centers both to reduce the risk of transmission of COVID-19 to healthcare workers and patients and to lessen the pressure on these services.

\section{General Principles}

- Assessment of patients should take account of patient and staff safety, the best interests of the patient, professional judgment, local urgent dental care center arrangements, and prioritization of the most urgent care needs.

- During the assessment, each patient's COVID-19 status must be established and recorded using the health board or local health system protocol. This will determine how care is managed at the designated urgent dental care centers if referral is required.

- Primary care dental triage should focus on the provision of the three A's: advice, analgesia, and antimicrobial.

o Patients should be advised that treatment options are severely restricted at this time and to call back in 48 to 72 hours if their symptoms have not resolved.

- It is advisable to liaise with local pharmacy colleagues to ensure that the products you might be recommending are available to your patients.

- Dental conditions that require urgent dental care should be referred via local pathways to the designated urgent dental care center following local protocols.

- Appropriate records should be kept of all patient contacts, including care management and onward referrals.

- Referrals should follow local handover guidelines and templates. $^{18}$

\section{Conclusion}

This new virus outbreak has challenged the economic, medical, and public health infrastructure of the world. Time alone will tell how the virus will impact our lives here in India. More so, future outbreaks of viruses and pathogens of zoonotic origin are likely to continue. Therefore, apart from curbing this outbreak, efforts should be made to devise comprehensive measures to prevent future outbreaks of zoonotic origin. The protocol mentioned in the review is based on the information available to date and might change with further ongoing investigations.

\section{References}

1. Navas-Martin SR, Weiss S. Coronavirus replication and pathogenesis: implications for the recent outbreak of severe acute respiratory syndrome (SARS), and the challenge for vaccine development. J Neurovirol 2004;10(2):75-85. DOI: 10.1080/13550280490280292.

2. Drexler JF, Gloza-Rausch F, Glende J, et al. Genomic characterization of severe acute respiratory syndrome-related coronavirus in European bats and classification of coronaviruses based on partial RNA-dependent RNA polymerase gene sequences. J Virol 2010;84(21):11336-11349. DOI: 10.1128/JVI.00650-10.

3. Yin Y, Wunderink RG. MERS, SARS and other coronaviruses as causes of pneumonia. Respirology 2018;23(2):130-137. DOI: 10.1111/ resp.13196.

4. Sahin AR, Erdogan A, Mutlu Agaoglu P, et al. 2019 Novel coronavirus (COVID-19) outbreak: a review of the current literature. EJMO 2020;4(1):1-7. DOI: 10.14744/ejmo.2020.12220.

5. Zaki AM, van Boheemen S, Bestebroer TM, et al. Isolation of a novel coronavirus from a man with pneumonia in Saudi Arabia. N Engl J Med 2012;367(19):1814-1820. DOI: 10.1056/NEJMoa1211721.

6. Imperial College London. Report 2: estimating the potential total number of novel coronavirus cases in Wuhan City, China. Jan 2020. Available at: https://www.imperial.ac.uk/mrcglobalinfectiousdisease-analysis/news--wuhan-coronavirus.

7. World Health Organization, 2019-nCoV Situation Report-22 on 12 February, 2020. https://www.who.int/docs/defaultsource/ coronaviruse/situation-reports/.

8. Xinhua. China's CDC detects a large number of new coronaviruses in the South China seafood market in Wuhan. Available at: https://www.xinhuanet.com/2020-01/27/c_1125504355.htm, Accessed 20 Feb 2020

9. Chen Z, Zhang W, Lu Y, et al. From SARS-CoV to Wuhan 2019-nCoV outbreak: similarity of early epidemic and prediction of future trends: Cell Press 2020. DOI: 10.1101/2020.01.24.919241.

10. Coronavirinae in ViralZone. Available at: https://viralzone.expasy. org/785. Accessed on 05 February 2019.

11. Subissi L, Posthuma CC, Collet A, et al. One severe acute respiratory syndrome coronavirus protein complex integrates processive RNA polymerase and exonuclease activities. Proc Natl Acad Sci U S A 2014;111(37):E3900-E3909. DOI: 10.1073/pnas.1323705111.

12. Chu H, Zhou J, Wong BH, et al. Productive replication of Middle East respiratory syndrome coronavirus in monocyte-derived dendritic cells modulates innate immune response. Virology 2014;454455:197-205. DOI: 10.1016/j.virol.2014.02.018.

13. WHO. Emergencies preparedness, response. Pneumonia of unknown origin-China. Disease outbreak news. Available at: https://www.who. int/csr/don/12-january-2020-novel-coronavirus-china/en/. Accessed on 05 February 2020.

14. Rothe C, Schunk M, Sothmann P, et al. Transmission of 2019-nCoV Infection from an asymptomatic contact in Germany, 30.01.2020. Available at: https://www.nejm.org/doi/full/10.1056/NEJMc2001468.

15. Tanu S. A review of coronavirus disease-2019 (COVID-19). Indian J Pediatr 2020;87(4):281-286. DOI: 10.1007/s12098-020-03263-6.

16. Chang $\mathrm{D}, \mathrm{Xu} \mathrm{H}$, Rebaza A. Protecting health-care workers from subclinical coronavirus infection. Lancet Respir Med 2020;8(3):e13. DOI: $10.1016 /$ S2213-2600(20)30066-7.

17. Li J, Li J, Xie X, et al. Game consumption and the 2019 novel coronavirus. Lancet Infect Dis 2020;20(3):275-276. DOI: 10.1016/ S1473-3099(20)30063-3.

18. Management of acute dental problems during COVID-19 pandemic, Scottish dental clinical effectiveness program. 30 March 2020. 